\title{
A household food inventory for North American Chinese
}

\author{
Jessie A Satia ${ }^{1, \star}$, Ruth E Patterson ${ }^{1}$, Alan R Kristal ${ }^{1}$, T Gregory Hislop ${ }^{2}$ \\ and Michele Pineda ${ }^{3}$ \\ ${ }^{1}$ Fred Hutchinson Cancer Research Center, Cancer Prevention Research Program, 1100 Fairview Avenue N., \\ MP-702, Seattle, WA 98109-1024, USA: ${ }^{2}$ British Columbia Cancer Agency, 600 West Tenth Avenue, Vancouver, \\ B.C. V5Z 4E6, Canada: ${ }^{3}$ Ministry of Health, Republic of Palau, P.O. Box 1714-T101, Koror PW 96940, Palau
}

Submitted 6 July 2000: Accepted 11 September 2000

\begin{abstract}
Objective: To determine whether a short set of questions about foods in the household can provide information about the fat-related dietary behaviour of individual household members in less-acculturated Chinese populations.

Design: Cross-sectional survey.

Participants: The study population included 244 adult females of Chinese ethnicity in Seattle, WA, and Vancouver, BC, Canada.

Setting: Bilingual interviewers collected information on the presence of 14 high-fat foods and seven reduced-fat foods in the household. Respondents were also asked about the consumption of foods and behaviour reflective of adoption of Western dietary practices, fat-related dietary behaviour, changes in consumption of high-fat foods since immigration, and sociodemographic characteristics.

Results: Although this was a less-acculturated sample, many households had Western foods such as butter (58\%), lunchmeats (36\%), snack chips (43\%), and 1\% or skim milk (48\%). Households with respondents who were younger, married, employed outside the home, and lived with young children had significantly more high-fat foods, while high education and longer percentage of life in North America were significantly associated with having more reduced-fat foods $(P \leq 0.05)$. Participants living in households with more high-fat foods had higher-fat dietary behaviour than those with fewer high-fat foods (fat-related dietary behaviour score, 1.54 versus 1.28; $P<0.001$ ). Women in households with more reduced-fat foods had a significantly decreased consumption of high-fat foods since immigration compared with those in households with fewer reduced-fat foods $(P<0.001)$. Western dietary acculturation was higher among women in households both with more high-fat foods and more reduced-fat food counterparts $(P \leq 0.05)$.

Conclusions: Our inventory of household foods was strongly associated with current dietary behaviour, changes in food consumption, and westernization of dietary patterns. This simple, practical measure may be a useful alternative dietary assessment tool in less-acculturated Chinese populations.
\end{abstract}

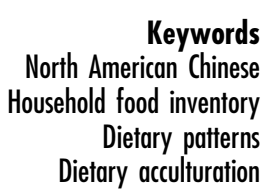

One of the Healthy People 2010 objectives is to improve the health status of minority populations. Asian Americans are one of the fastest growing minority groups in North America (US and Canada), and ethnic Chinese are the largest Asian subgroup ${ }^{1}$. Compared with Chinese living in Asia, those in North America have higher rates of several chronic diseases, including cardiovascular disease and some cancers ${ }^{2,3}$. These increased risks in North Americandwelling Chinese have been largely attributed to adoption of Western dietary behaviour, in particular diets high in fat and low in fruits and vegetables ${ }^{2-6}$. Therefore, health programmes, such as nutrition interventions designed to encourage maintenance of a traditional Chinese diet, could play an important role in meeting the Healthy People goals.

The effects of nutrition interventions are most often evaluated based on changes in self-reported dietary behaviour $^{7,8}$. However, dietary assessment is usually a significant methodological challenge and measuring dietary intake in Chinese populations is further complicated by a number of factors. Many Chinese immigrants are linguistically isolated and may therefore be unable to complete dietary assessment instruments. Meals are usually consumed in mixed dishes and several people 
may eat from the same serving dish ('fire pot'), so respondents may have difficulty reporting intake of individual foods 9 . Chinese do not typically measure food and may have difficulty estimating serving sizes (personal communication, Marion Lee, May 1998). Finally, Chinese immigrants may not be familiar with concepts such as 'servings per day'. Therefore, self-reported diet may not be an appropriate approach to assessing food intake in less-acculturated Chinese immigrants. These difficulties motivate the development of simple, practical and valid dietary assessment tools for this population.

Environmental measures that reflect dietary behaviour, such as those that assess characteristics of communities or the physical environment, may be useful indicators of dietary intake and dietary change. For example, there is good evidence that supermarket shelf space can be used as a measure of diet in the community ${ }^{10}$. Similarly, Patterson et al. showed that availability of high-fat foods in the households of a random sample of Washington State residents is related to dietary behaviour ${ }^{11}$. At the practical level, these measures are fairly easy to administer and are appropriate for low-literacy individuals ${ }^{11}$. Therefore, environmental indicators of diet may be useful dietary assessment and intervention evaluation tools for less-acculturated Chinese.

Our objective was to determine whether foods in the household can provide useful information about the dietary patterns of individual household members in lessacculturated North American Chinese. Specifically, we wanted to ascertain whether the presence of high-fat and reduced-fat foods in the household can be a meaningful indicator of an individual's fat-related dietary patterns. To meet this aim, we examined associations between the number of high-fat and reduced-fat foods in the home and key factors related to dietary behaviour in immigrant populations: sociodemographic characteristics, Western dietary acculturation, dietary change, and current diet.

\section{Methods}

\section{Design and participants}

The data reported here were collected as part of the Chinese Women's Health Project, a study examining cervical cancer screening practices in women of Chinese descent aged 20 years and older living in Seattle, WA, and Vancouver, BC, Canada. This research received human subjects review approval.

Details of the survey procedures have been published ${ }^{12}$. In brief, participants in each city were selected by random sampling from the Chinese female population in geographically defined catchment areas where a high proportion (20-63\%) of residents are Chinese ${ }^{13,14}$. We used various sources (including a commercially available listing, published articles, and cancer registry data) to identify Chinese households within our catchment areas and compile a list of Chinese surnames ${ }^{15}$. Surnames that appeared in two or more sources were matched to 1998 telephone book listings in Seattle and Vancouver to provide a sampling population from each city.

Interviews were conducted during 1999 by trained bilingual (Cantonese-English and Mandarin-English) female interviewers recruited from local Chinese communities. Interviews were administered in person at the participant's home in the language of her choice. Up to five in-person attempts were made at contacting each household, and only one woman per household was interviewed. Participants received \$10 or \$15 (Canadian) to acknowledge appreciation of their participation.

For the main study, data were collected on sociodemographic characteristics, acculturation, cervical cancer screening practices, and health care-related issues. All survey questions were developed in English, translated into Chinese, back-translated, reconciled, and pre-tested. The estimated response rate for the main study in both cities was 64\%. For this ancillary study, dietary data were collected on $17 \%$ of the participants in the main study using a stratified random sampling procedure.

\section{Housebold food inventory}

The household food inventory consists of 14 foods (Table 1) that we selected using three main sources of information. First, we used data collected from 30 qualitative interviews and two focus groups with lessacculturated Chinese women in Seattle, WA. Details of our qualitative methods have been published ${ }^{16}$. In brief, during the qualitative research, participants were asked to list the foods they had in their home (including meats, dairy products, and savoury snacks) and whether they identified these foods as 'Chinese' or 'American'. Next, we compared the foods listed against (i) published data on the major contributors of total fat in North American and Chinese diets ${ }^{11,17-19}$, and (ii) data from other studies that have found these foods to be useful indicators of dietary change ${ }^{5,6,20}$. Food items listed by qualitative participants that are major contributors of fat and/or indicator foods of dietary change were included in the household food inventory.

For this survey, respondents were asked whether (yes or no) these foods are currently in their household, including in the pantry, cabinets, and refrigerators. For seven foods (cheese, milk, mayonnaise, salad dressing, cookies, cakes and pies, and snack chips), we also ascertained whether the participants had the reduced- or non-fat version.

We divided the items in the household inventory into two scales: a 14-item 'high-fat' and a seven-item 'reducedfat' household food inventory. For analysis purposes, the items in the two scales were coded as 0 or 1 , where a higher score indicates more high-fat and more reduced-fat foods in the household, respectively. A summary score for each scale was calculated as the mean of the non-missing items. We also divided the household food inventory 
Table 1 Percent of respondents reporting items in a household food inventory in a study of Chinese American and Chinese Canadian women $(n=244)$

\begin{tabular}{lc}
\hline Scale item & Percent responding \\
(Do you have this food item in your house right now...) & yes \\
\hline 'High-fat household inventory items & 91 \\
Beef, pork, or lamb & 68 \\
Nuts or peanut butter & 60 \\
Regular cookies & 58 \\
Butter or margarine & 44 \\
$2 \%$ milk & 43 \\
Regular (full-fat) cheese & 43 \\
Regular potato, corn, or tortilla chips & 36 \\
Regular lunch meats & 29 \\
Regular mayonnaise & 27 \\
Whole milk & 25 \\
Regular salad dressing & 25 \\
Regular cakes or pies & 16 \\
Coconut milk & 7 \\
Bacon or Chinese sausage & \\
Total 'high-fat' household food inventory scale* (Kuder-Richardson 20 & $0.72)$ \\
'Reduced-fat household inventory items & 48 \\
1\% or skim milk & 22 \\
Reduced-fat or non-fat cookies & 21 \\
Reduced-fat or non-fat salad dressing & 20 \\
Reduced- or non-fat cheese & 18 \\
Reduced-fat or non-fat mayonnaise & 16 \\
Reduced-fat or non-fat potato, corn, or tortilla chips & 5 \\
Reduced-fat or non-fat cakes or pies & \\
Total 'reduced-fat' household food inventory scale† (Kuder-Richardson 20 & \\
* Items coded such that a higher score indicates more high-fat foods in the home. \\
† Items coded such that a higher score indicates more reduced-fat foods in the home. & \\
\hline
\end{tabular}

scores approximately into tertiles of low, intermediate and high. The summary scales had fair to good internal consistency (Kuder-Richardson 20 coefficient $=0.57$ for the reduced-fat scale and 0.72 for the high-fat scale). One item (organ meats) was dropped from further analyses, because of its low correlation with the summary score.

\section{Dietary assessment}

\section{Fat-related dietary behaviour}

Fat-related dietary behaviour was assessed using a version of the Fat-Related Diet Habits Questionnaire (DHQ) that we modified slightly to reflect Chinese eating patterns. The development and validation of the DHQ have been published $^{21}$. Our 13-item questionnaire asked about diet over the past month and assessed avoiding fat in cooking, avoiding deep-fried foods, boiling or steaming instead of stir-frying, modifying meats to be lower in fat, and substituting lower-fat alternatives for high-fat foods. Response options were 'always/usually', 'sometimes', and 'rarely/never', and were coded 1 to 3 so that a higher score corresponds to higher-fat dietary behaviour. The summary score was calculated as the mean of the nonmissing items. In a recent validation study, the correlation of the DHQ summary score with percent energy from fat from a 94-item food frequency questionnaire was $0.53^{21}$.
Changes in consumption of high-fat foods

Assessment of changes in the consumption of high-fat foods was modelled on a validated approach described by Jenkins et al. for Vietnamese populations ${ }^{22}$. Our sixitem instrument assessed changes in consumption of foods that are strongly associated with (i) either the risk of cancer and other chronic diseases or (ii) westernization of dietary patterns. Participants were asked whether consumption of meats, fish, and tofu had increased, decreased, or remained the same during the last 5 years or since immigration to North America (if the participant had lived in North America for less than 5 years). Items were coded 1 to 3 so a higher score corresponds to increased consumption of high-fat foods since immigration. Each participant's summary score was computed as the mean of the non-missing values.

\section{Western dietary acculturation}

Western dietary acculturation is defined as the process by which immigrant groups adopt Western dietary behaviour $^{23}$, and was assessed using a scale that has been validated in this population. Details on the development and validation of this instrument have been published ${ }^{24}$. Briefly, items in the 10-item Western dietary acculturation scale were selected using data from 30 qualitative interviews and two focus groups with women of Chinese descent living in Seattle, WA ${ }^{16}$. During this qualitative research, we elicited information on participants' dietary 
habits, and used this information to identify indicator foods and dietary behaviour associated with westernization of dietary patterns. Examples of items include snacking between meals, eating at fast-food restaurants, and eating dairy products. Response options were yes or no, based on the respondent's dietary practices in the past month. For analysis purposes, items were coded as 0 or 1 , with a higher score correlating positively with the degree of Western acculturation. A respondent's summary score was computed as the mean of the non-missing responses. The summary scale had good internal consistency (Kuder-Richardson 20 coefficient $=0.72$ ).

\section{Statistical analyses}

We conducted data analyses using SAS 6.12 (SAS version 6, first edition, SAS Institute Inc., Cary, NC, 1993). Analyses of variance were used to test for significant differences in the mean values of household food inventory scores between subgroups of sociodemographic variables, and post-hoc Duncan's multiple range tests assessed which groups differed significantly from each other. Linear regression was used to examine associations of dietary measures with the household food inventory scales, controlling for age, education, and city of residence. Pearson's correlation coefficients measured associations among continuous variables.

\section{Results}

There were 113 participants from Seattle and 131 from Vancouver, with a mean age of $52.4 \pm 14.3$ years. A quarter of the respondents had more than high school education and about half were employed outside the home. About 80\% were married and owned their homes and almost all were born outside North America.

Table 1 gives the frequency of the responses to items in the household food inventory scales. Although this was a less-acculturated sample, about $40-50 \%$ of households had 'Western' foods such as butter or margarine, snack chips and 2\% milk in their homes. Only 30-40\% of respondents lived in homes with lunchmeats and mayonnaise. Less than a quarter of households had reduced-fat cakes and pies, cheese and cookies, but almost half had $1 \%$ or skim milk.

Table 2 gives the mean household food inventory scores of each scale stratified by sociodemographic characteristics. There were significantly more high-fat foods in households containing respondents who were

Table 2 Mean household food inventory scores of participants by sociodemographic characteristics in a study of Chinese American and Chinese Canadian Women $(n=244)$

\begin{tabular}{|c|c|c|c|}
\hline \multirow[b]{2}{*}{ Characteristic } & \multirow[b]{2}{*}{$n(\%)$} & \multicolumn{2}{|c|}{ Mean household food inventory score* } \\
\hline & & 'High-fat'† & 'Reduced-fat'‡ \\
\hline Total & $244(100 \%)$ & $2.07 \pm 0.81 \S$ & $1.80 \pm 0.73 \S$ \\
\hline \multicolumn{4}{|l|}{ Age (years) } \\
\hline $20-39$ & $52(21 \%)$ & $2.33^{a}$ & 1.83 \\
\hline $40-55$ & $95(39 \%)$ & $2.21^{\mathrm{a}}$ & 1.83 \\
\hline$>55$ & $97(40 \%)$ & $1.82^{b}$ & 1.75 \\
\hline$P$ for trend & & 0.0001 & \\
\hline \multicolumn{4}{|c|}{ Education (years) } \\
\hline$\leq 6$ & $69(28 \%)$ & 2.01 & $1.59^{\mathrm{a}}$ \\
\hline $7-12$ & $123(51 \%)$ & 2.09 & $1.84^{\mathrm{b}}$ \\
\hline$\geq 13$ & $52(21 \%)$ & 2.14 & $1.98^{\mathrm{b}}$ \\
\hline$P$ for trend & & & 0.004 \\
\hline \multicolumn{4}{|l|}{ Marital status } \\
\hline Married & $193(79 \%)$ & $2.16^{\mathrm{a}}$ & 1.82 \\
\hline Not married & $51(21 \%)$ & $1.71^{\mathrm{b}}$ & 1.72 \\
\hline$P$ for trend & & 0.0001 & \\
\hline \multicolumn{4}{|c|}{ Live with children $6-18$ years } \\
\hline Yes & $85(35 \%)$ & $2.44^{\mathrm{a}}$ & 1.87 \\
\hline No & $159(65 \%)$ & $1.86^{\mathrm{b}}$ & 1.75 \\
\hline$P$ for trend $\emptyset$ & & 0.0001 & \\
\hline \multicolumn{4}{|c|}{ Employed outside the home } \\
\hline Yes & $118(48 \%)$ & $2.21^{a}$ & 1.84 \\
\hline No & $126(52 \%)$ & $1.93^{b}$ & 1.76 \\
\hline$P$ for trend $\emptyset$ & & 0.0001 & \\
\hline \multicolumn{4}{|c|}{ Percentage of life spent in North America (\%) } \\
\hline$<20$ & $90(37 \%)$ & 2.04 & $1.63^{\mathrm{a}}$ \\
\hline $20-39$ & $92(38 \%)$ & 2.06 & $1.81^{\mathrm{a}, \mathrm{b}}$ \\
\hline $40-100$ & $62(25 \%)$ & 2.15 & $1.92^{\mathrm{b}}$ \\
\hline$P$ for trend $\emptyset$ & & & 0.02 \\
\hline
\end{tabular}

* Means with the same superscripts are not significantly different.

† Items coded such that a higher score indicates more high-fat foods in the home.

$\ddagger$ Items coded such that a higher score indicates the presence of more reduced-fat foods in the home.

$\S$ Mean \pm SD

ๆ Only $P$ values $\leq 0.05$ are shown 
Table 3 Associations of household food inventory scales with criterion measures in a study of Chinese American and Chinese Canadian women $(n=244)$

\begin{tabular}{|c|c|c|c|c|c|c|c|}
\hline \multirow[b]{3}{*}{ Household food inventory scales } & \multirow[b]{3}{*}{$n(\%)$} & \multicolumn{6}{|c|}{ Criterion measures* } \\
\hline & & \multicolumn{2}{|c|}{$\begin{array}{l}\text { Mean fat-related } \\
\text { dietary behaviour } \\
\text { score } \dagger\end{array}$} & \multicolumn{2}{|c|}{$\begin{array}{l}\text { Mean fat-related } \\
\text { change } \\
\text { score } \neq\end{array}$} & \multicolumn{2}{|c|}{$\begin{array}{c}\text { Mean Western } \\
\text { dietary acculturation } \\
\text { score§ }\end{array}$} \\
\hline & & Raw & Adj. ${ }^{* *}$ & Raw & Adj. ${ }^{* *}$ & Raw & Adj. ** \\
\hline & $244(100 \%)$ & $1.39 \pm 0.34 \emptyset$ & & $2.21 \pm 0.379$ & & $0.61 \pm 0.24 \rrbracket$ & \\
\hline \multicolumn{8}{|l|}{ 'High-fat' household food inventory score†† } \\
\hline Low (none or few high-fat foods) & $72(30 \%)$ & $1.24^{\mathrm{a}}$ & $1.28^{\mathrm{a}}$ & 2.16 & 2.17 & $1.62^{\mathrm{a}}$ & $1.77^{\mathrm{a}}$ \\
\hline Intermediate (some high-fat foods) & $83(34 \%)$ & $1.33^{\mathrm{a}}$ & $1.32^{\mathrm{a}}$ & 2.25 & 2.25 & $2.16^{\mathrm{a}}$ & $2.13^{\mathrm{b}}$ \\
\hline High (more high-fat foods) & $89(36 \%)$ & $1.56^{\mathrm{b}}$ & $1.54^{\mathrm{b}}$ & 2.25 & 2.23 & $2.37^{b}$ & $2.33^{\mathrm{b}}$ \\
\hline$P$ for trend & & 0.0001 & 0.0001 & 0.39 & 0.16 & 0.0001 & 0.0001 \\
\hline \multicolumn{8}{|c|}{ 'Reduced-fat' household food inventory scoreł‡ } \\
\hline Low (none or few reduced-fat foods) & $95(39 \%)$ & 1.40 & 1.42 & $2.31^{a}$ & $2.33^{\mathrm{a}}$ & $1.91^{a}$ & $1.97^{\mathrm{a}}$ \\
\hline Intermediate (some reduced-fat foods) & $86(35 \%)$ & 1.37 & 1.38 & $2.23^{a, b}$ & $2.20^{\mathrm{a}, \mathrm{b}}$ & $2.13^{a, b}$ & $2.15^{\mathrm{a}, \mathrm{b}}$ \\
\hline High (more reduced-fat foods) & $63(26 \%)$ & 1.39 & 1.37 & $2.16^{\mathrm{b}}$ & $2.17^{\mathrm{b}}$ & $2.24^{\mathrm{b}}$ & $2.22^{\mathrm{b}}$ \\
\hline$P$ for trend & & 0.90 & 0.45 & 0.02 & 0.02 & 0.02 & 0.04 \\
\hline
\end{tabular}

* Means with the same superscripts are not significantly different.

† Fat-related dietary behaviour score coded such that a high score indicates high-fat dietary behaviour.

‡ Fat-related change score coded such that a high score indicates increase in consumption of high-fat foods after immigration.

$\S$ Western Dietary Acculturation Scale coded such that a high score indicates more Western dietary acculturation.

Il Mean \pm SD.

** Adjusted for age, education, and city of residence.

t† Items coded such that a higher score indicates more high-fat foods in the home.

拉 Items coded such that a higher score indicates more reduced-fat foods in the home.

younger, married, employed outside the home, and lived with young children $(P<0.05)$. There were significantly more reduced-fat foods in households with highly educated women who had spent a longer percentage of their lives in North America $(P<0.05)$.

Table 3 gives raw and adjusted associations of the household food inventory scales with the dietary measures. In both raw and adjusted analyses, respondents living in households with more high-fat foods had higher fat-related dietary behaviour compared with those with fewer high-fat foods (fat-related dietary behaviour score $=1.54$ versus 1.28, $P<0.001$ ). Participants in households with more reduced-fat foods had significantly decreased consumption of high-fat foods since immigration compared with those who had fewer reduced-fat foods (fat-related change score $=2.17$ versus $2.33, P=$ 0.02). Adoption of Western dietary practices (i.e. Western dietary acculturation) was positively associated both with having more high-fat foods and more reduced-fat foods in the home (both $P<0.05$ ).

Table 4 gives correlations of the household food inventory scales with sociodemographic characteristics and the criterion measures. There were good correlations (Pearson's $r$ ) between the high-fat household food inventory scale and the high-fat dietary behaviour score (0.40) and Western dietary acculturation (0.42). Correlations with the reduced-fat scale were -0.15 for change in consumption of high-fat foods and 0.17 for Western dietary acculturation. All correlations were statistically significant $(P<0.05)$. Controlling for sociodemographic characteristics did not change these correlations appreciably.

\section{Discussion}

This brief household food inventory was significantly associated with diet, acculturation, and sociodemographic characteristics. Respondents living in households with more high-fat foods were more likely to be younger, married, live with young children, and to be employed outside the home; while those with more reduced-fat foods were more highly educated and had lived in North America the longest. Having high-fat foods in the home was significantly associated with high-fat dietary behaviour, while having more reduced-fat foods was associated with decreased consumption of high-fat foods after immigration. Western dietary acculturation correlated positively with having both more high- and reduced-fat foods. These associations among dietary, sociodemographic and acculturation characteristics are consistent with findings from other studies in Chinese immigrants, which generally indicate that fat intake increases with Western acculturation and affluence ${ }^{6,25,26}$. To our knowledge, the finding of more reduced-fat foods in households with Western acculturated respondents has not been previously reported. This association is, however, not entirely unexpected because since the traditional Chinese diet typically emphasizes whole foods ${ }^{2,5,27}$, using reduced-fat products is more likely to be an adopted Western (than Chinese) behaviour.

Although this was a less-acculturated sample, many households contained foods that are not typically associated with a traditional Chinese diet, such as savoury snacks, deli meats, and skim milk. These findings suggest that dietary assessment instruments for Chinese 
Table 4 Correlations of the household food inventory scales with sociodemographic characteristics and criterion measures in a study of Chinese American and Chinese Canadian women $(n=244)$

\begin{tabular}{llc}
\hline & \multicolumn{1}{c}{ Pearson's $r(P$ value $)$} \\
\cline { 2 - 3 } $\begin{array}{l}\text { 'High-fat' household } \\
\text { food inventory scale }\end{array}$ & $\begin{array}{c}\text { 'Reduced-fat' household } \\
\text { food inventory scale† }\end{array}$ \\
\hline $\begin{array}{l}\text { Sociodemographic characteristic } \\
\text { Age }\end{array}$ & $-0.31(0.0001) \ddagger$ & -0.07 \\
Education & 0.10 & $0.25(0.0001)$ \\
Marital Status & $-0.24(0.0001)$ & -0.08 \\
Live with children 6-18 years & $-0.33(0.0001)$ & -0.10 \\
Employment outside the home & $-0.17(0.0090)$ & -0.08 \\
Proportion of life spent in North America & 0.05 & $0.16(0.02)$ \\
Criterion measures & $0.40(0.0001)$ & 0.02 \\
Fat-related dietary behaviour score & 0.10 & $-0.15(0.02)$ \\
Fat-related change score & $0.42(0.0001)$ & $0.17(0.007)$ \\
\hline Western dietary acculturation score &
\end{tabular}

* Items coded such that a higher score indicates more high-fat foods in the home.

$\dagger$ Items coded such that a higher score indicates more reduced-fat foods in the home.

$\ddagger$ Only $P$ values $\leq 0.05$ are shown.

populations that don't ask about Western foods will miss significant sources of fat.

Our findings are consistent with other studies that have examined the usefulness of environmental measures of the food supply in assessing dietary intake. For example, in a random-digit-dial survey of 1002 participants, Patterson et al. found that individuals with low-fat pantries consumed $32 \%$ of energy from fat, compared with $37 \%$ for those with high-fat pantries ${ }^{11}$. In a study of 193 households in New York State, Kendall et al.reported a significant decrease in the frequency of fruit and vegetable consumption as well as greater individuallevel measures of food insecurity among women living in households with poor food availability ${ }^{28}$.

Given the challenges of assessing diet, the household food inventory presents a number of advantages over other dietary assessment instruments for Chinese populations: it is brief, objective, easy to administer and analyse, has low participant burden, is appropriate for low-literacy individuals, and is less susceptible to social desirability bias. In addition, these scales may be sensitive enough to detect changes in dietary behaviour, since they were significantly associated with the dietary acculturation and change in food consumption measures.

This study has a number of limitations. We had a relatively small sample, comprised primarily of lessacculturated women, which may limit the generalizability of the findings. There may be differences between survey respondents and non-respondents that can bias the results. These data are also based on self-report, which may not be accurate. A major limitation is that our dietary measures (i.e. fat-related dietary behaviour and changes in high-fat food consumption) could not precisely estimate fat intake. Multiple food records or food frequency questionnaires, which are more comprehensive, generally yield more accurate estimates of fat intake. Validation of the household food inventory using more precise assessment measures, such as percentage of energy from fat, would significantly improve its applicability for widespread research.

In spite of these limitations, this inventory of household foods was strongly associated with current dietary behaviour, changes in food consumption, and westernization of dietary patterns. This measure takes only a few minutes to administer and is easy for respondents to answer. Further, in theory, the household food inventory can provide information on the food intake of all household members ${ }^{11}$. Therefore, this simple, practical, environmental measure may be a useful alternative dietary assessment tool in nutrition intervention and education programs for less-acculturated Chinese populations.

\section{Acknowledgements}

We are grateful to The Chinese Women's Health Project Investigators, staff, and interviewers for their tireless efforts and contributions to this project.

\section{References}

1 US Bureau of the Census. Population Projections of the United States, by Age, Sex, Race, and Hispanic Origin: 1995 to 2050. Popul. Char Ser P-25 No. 1130. Washington DC: US Government Printing Office, 1996.

2 Whittemore AS, Kolonel LN, Wu AH, et al. Prostate cancer in 
relation to diet, physical activity, and body size in blacks, whites, and Asians in the United States and Canada. J. Natl. Cancer Inst. 1995; 87: 652-61.

3 Whittemore AS, Wu-Williams AM, Lee M. Diet, physical activity, and colorectal cancer among Chinese in North America and China. J. Natl. Cancer Inst. 1990; 82: 915-26.

4 Chen A, Ng P, Sam P. Special health problems of Asians and Pacific Islanders. In: Matzen RN, Long RS, eds. Clinical Preventive Medicine. St. Louis, MO: Mosby, 1993.

5 Lee MM, Wu-Williams A, Whittemore AS, et al. Comparison of dietary habits, physical activity, and body size among Chinese in North America and China. Int. J. Epidemiol. 1994; 23: 984-90.

6 Sun WY, Chen WW. A preliminary study of potential dietary risk factors for coronary heart disease among Chinese American adolescents. J. Sch. Health 1994; 64: 368-71.

7 Glanz K. Progress in dietary behavior change. Am. J. Health Promot. 1999; 14: 112-7.

8 Coates RJ, Bowen DJ, Kristal AR, et al. The Women's Health Trial Feasibility Study in minority populations: changes in dietary intakes. Am. J. Epidemiol. 1999; 149: 1104-12.

9 Lee MM, Lee F, Ladenla SW, Miike R. A semiquantitative dietary history questionnaire for Chinese Americans. Ann. Epidemiol. 1994; 4: 188-97.

10 Crocket SJ, Potter JD, Wright MS, Bacheller A. Validation of a self-reported shelf inventory to measure food purchase behavior. J. Am. Diet. Assoc. 1992; 92: 694-7.

11 Patterson RE, Kristal AR, Shannon J, Hunt JR, White E. Using a brief household food inventory as an environmental indicator of individual dietary practices. Am.J. Public Health 1997; 87: 272-5.

12 Do HH, Taylor VM, Yasui Y, Jackson JC, Tu SP. Cervical cancer screening among Chinese immigrants in Seattle, WA. J. Imm. Health in press.

13 US Department of Commerce. 1990 Census of Population and Housing: Population and Housing Characteristics for Census Tracts and Black Numbering Areas - Seattle, WA PMSA. US Department of Commerce: Washington, DC, 1993.

14 Canada Dominion Bureau of Statistics. Canadian Census of Population Documentation Information, 1996. Ottawa, Canada.
15 Choi B. Use of surnames to identify individuals of Chinese ancestry. Am. J. Epidemiol. 1993; 138: 723-34.

16 Satia JA, Patterson RE, Taylor VM, et al. Use of qualitative methods to study diet, acculturation, and health in Chinese American women. J. Am. Diet. Assoc. 2000; 100: 934-40.

17 Subar AF, Krebs-Smith SM, Cook A, Kahle LL. Dietary sources of nutrients among US adults 1989 to 1991. J. Am. Diet. Assoc. 1998; 98: 537-47.

18 Patterson RE, Kristal AR, Lynch JC, White E. Diet - cancer related beliefs, knowledge, norms, and their relationship to healthful diets. J. Nutr. Educ. 1995; 27: 86-92.

19 Tian HG, Nan Y, Liang XQ, et al. Relationship between serum lipids and dietary and non-dietary factors in a Chinese population. Eur. J. Clin. Nutr. 1995; 49: 871-82.

20 Patterson RE, Kristal AR, White E. Do beliefs, knowledge, and perceived norms about diet and cancer predict dietary change? Am. J. Public Health 1996; 86: 1394-400.

21 Shannon J, Kristal AR, Curry SJ, Beresford SA. Application of a behavioral approach to measuring dietary change: the fatand fiber-related diet behavior questionnaire. Cancer Epidemiol. Biomarkers Prev. 1997; 6: 355-61.

22 Jenkins CN, McPhee SJ, Bird JA, Bonilla NT. Cancer risks and prevention practices among Vietnamese refugees. West. J. Med. 1990; 153: 34-9.

23 Negy C, Woods DJ. The importance of acculturation in understanding research with Hispanic Americans. Hisp. J. Behav. Sci. 1992; 14: 224-47.

24 Satia JA, Patterson RE, Kristal AR, Hislop GT, Yasui Y, Taylor VM. Development of dietary acculturation scales for Chinese Americans and Chinese Canadians. J. Am. Diet. Assoc. in press.

25 Schultz JD, Spindler AA, Josephson RV. Diet and acculturation in Chinese women. J. Nutr. Educ. 1994; 26: 266-72.

26 Pan YL, Dixon Z, Himburg S, Huffman F. Asian students change their eating patterns after living in the United States. J. Am. Diet. Assoc. 1999; 99: 54-7.

27 Campbell TC, Chen J. Energy balance: interpretation of data from rural China. Toxicol. Sci. 1999; 52(2 Suppl.): 87-94.

28 Kendall A, Olson CM, Frongillo EA Jr, Validation of the Radimer/Cornell measures of hunger and food insecurity. $J$. Nutr. 1995; 125: 2793-801. 